Original Research Paper

\title{
Enhancing Social Science Research in the UAE: An Open Source Software Solution University of Sharjah (UOS) Case Study
}

\author{
${ }^{1}$ Manar Abu Talib, ${ }^{1}$ Muhammed AbuOdeh, ${ }^{2}$ Adhraa Almansoori and ${ }^{2}$ Arwa AlNauimi \\ ${ }^{1}$ Department of Computer Science, University of Sharjah, P.O.Box 27272, Sharjah, UAE \\ ${ }^{2}$ College of Technological Innovation, Zayed University, P.O. Box 4783, Abu Dhabi, UAE
}

\author{
Article history \\ Received: 11-05-2014 \\ Revised: 25-06-2014 \\ Accepted: 10-09-2014 \\ Corresponding Author: \\ Manar Abu Talib \\ Department of Computer Science, \\ University of Sharjah, P.O.Box \\ 27272, Sharjah, UAE \\ Email: manar.abutalib@gmail.com
}

\begin{abstract}
Researchers, practitioners and social scientists all agree on the enormous importance of social science research in advancing human knowledge and informing public policy makers. The National Research Foundation, along with other organizations in the UAE, is leading the initiative to integrate the efforts of social research institutions in order to support the decision making process in the UAE. As indicated by a number of social researchers during the last forum held on May 29-30, 2012, at the Emirates Center for Strategic Studies and Research, Abu Dhabi, UAE, there is a huge demand for a unified database for social science research in the UAE. In this study, we report on a survey that was distributed to more than 140 social science researchers in four main cities in the UAE: Abu Dhabi, Al Ain, Dubai and Sharjah. It shows that more than $90 \%$ of the researchers in the field of social sciences have faced challenges when conducting their research. We have also conducted interviews with the faculty members in the Department of Social Science at the University Of Sharjah (UOS). Although there are library resources available at UOS, researchers still face such challenges, compared to their colleagues at other universities. As a result, the Dataverse Network "Bayanatona", an open source application, was proposed as a complete solution for these researchers in the UAE.
\end{abstract}

Keywords: UAE, University of Sharjah, Social Science Research, Open Source Software, Dataverse, Ankabut, Harvard University

\section{Introduction}

The United Arab Emirates (UAE) is one of the most technologically advanced countries in the Gulf region and the Middle East, with its state-of-the-art telecommunications infrastructure (ESCWA, 2011). Most homes have access to many of the communications technologies that are available in western countries. According to the latest survey by the World Economic Forum, The Global Information Technology Report 2013, the UAE now ranks 25th in the world on The Networked Readiness Index (WEF,
2013). In the UAE, it is anticipated that spending on software will reach USD \$843 million in 2013 (UAEITR, 2013). A 2009 survey conducted by the International Data Corporation (IDC) found that the Open Source Software (OSS) market has experienced a strong boost from the current economic downturn, with worldwide revenues expected to grow at a compound annual growth rate of $22.4 \%$, to reach $\$ 8.1$ billion by 2013 (Jaspersoft, 2010). This expected growth is also attributed to the increased quality, reliability and support services supplied by OSS providers. In a difficult economic climate, most IT departments are 
under increased scrutiny and pressure to reduce costs. As a result, many are turning to OSS providers.

Currently, many information systems in the UAE are proprietary, as they are elsewhere in the world and require extensive customization that only specific vendors can perform, due to copyright, licensing and patent constraints. Most organizations allocate a substantial amount of time and money to software debugging, maintenance and upgrading by adding new functionalities and sometimes even redefining their goals and priorities. Faced with shrinking financial resources, some academic and research organizations have already turned to OSS to fulfill their information and technological needs. OSS is also beneficial to law enforcement agencies and to research and development, defense and legal and justice departments to meet their intrinsically stringent security and privacy requirements. This openly available software is developed in the technological community as an alternative to the proprietary software owned by corporations WEBOPEDIA, 2013. Our literature survey shows that there is no real OSS development or deployment strategy in place in developing countries, in comparison to those found in developed countries.

\section{Literature Review}

\section{Open Source Software (OSS)}

Open Source Software (OSS) is defined as software, the source code of which is available to all users free of charge. It is very often developed in a public, collaborative manner, motivated by an altruistic desire to improve society at large: Society comes first and individual commerce a distant second. "The promise of open source is better quality, higher reliability, more flexibility, lower cost and an end to predatory vendor lock-in." (OSI, 2013). In the UAE, OSS could be a valuable way to avoid total dependence on a single supplier or on foreign providers, with a view to further strengthening national security. It also opens the door to customized applications designed to develop appropriate information systems that best fit the needs of the organization. Moreover, it could support sustainability and provide an opportunity to UAE undergraduate/graduate students, researchers and developers to enhance their programming skills. OSS complements the strategic planning goals of the UAE in terms of cost-effectiveness and increased accessibility to knowledge. With it, managers could experiment with the suitability of business solutions without paying licensing fees. For example, many managers are already turning to OSS applications as viable sourcing alternatives for databases, data warehouses and enterprise-grade software applications (such as Enterprise Resource Planning-ERP) to reduce costs and increase competitive advantage. The cost of a desktop is not a major issue; however, having a valid license for software can be a real financial concern. The use of OSS would also eliminate the practice of "cracking" software, a term that has no meaning in the OSS community.

OSS is not limited to particular research tools, experimental toolkits, or visualization plug-ins. A wide range of system and application software can be envisaged, including programming languages, databases, information rendering and visualization tools, operating systems, applets, business applications, digital forensic tools, simulations tools and privacy and security toolkits. These applications are also designed to run on tiny mobile phones, enterprise level database servers and intrusion detection systems.

Several researchers have looked at the advantages and disadvantages of OSS. A complete list is outside the scope of this study. Below is a list of the most important of them

\section{Advantages:}

- Lower development and maintenance costs

- Freedom to choose maintenance contractors

- Less dependence on a single service provider

- Frequent releases and updates

- Easy and enriched customization

- A viable alternative when replacing legacy software

- Reusable older legacy hardware

- The opportunity to develop appropriate solutions that best meet organizational needs

Disadvantages:

- No user manual available

- Lack of technical support

- Missing links in the infrastructure

- Lack of OSS applications

- Difficulty in migrating to OSS due to high degree of reliance on commercial products

\section{Data Verse Network (DVN)}

The Data Verse Network (DVN) is an open source application designed to publish, share, reference, 
extract and analyze research data. It is an online digital repository for social science research data (Crosas, 2011). The DVN provides a central repository infrastructure for professionally archiving backups, data for recovery purposes and standardsbased persistent identifiers, as well as addressing data fixity and metadata storage, conversion and preservation and standards-based persistent identifiers, data fixity and metadata storage, conversion and preservation (Crosas, 2011). As well, the DVN facilitates data availability to other researchers and enables those researchers to replicate the work of others.

Development of the DVN started in 2006 at the Institute for Quantitative Social Science (IQSS) at Harvard University (Crosas, 2011). When it was released in 2007, the DVN hosted hundreds of virtual archives, provided access to more than 37,000 studies and constituted more than 600,000 files that were distributed across hundreds of dataverses (Crosas, 2011).

Moreover, the DVN has a number of significant features, such as: Interface simplicity, scalability and variety, user friendliness, an interactive environment and manageable data and privileges. The DVN is simple to use and doesn't require advanced skills or background in information technology. Figure 1 shows the homepage of our installed DVN, which includes various dataverses (universities, in this case). The first letter of a specific dataverse can be used to search the DVN more effectively.

A dataverse can be a university, a journal, or a research institution, for example. It is easy for both administrators and contributors to upload studies and equally easy for them to view without a guide or manual. Moreover, the DVN environment is interactive, in that it provides users with the ability to give feedback on the studies uploaded to it. The DVN also allows different privileges and permissions for the administrators, users and contributors of each dataverse, for example:

- Network Admin: Can add and remove new administrators, contributors and curators; can create and edit dataverses and studies

- Sub Admin: Can add and remove new administrators, contributors and curators, but cannot modify the role of the network admin; Can edit his/her dataverse (s) and all the studies

- Contributor: Can create, edit and remove studies

- User: Can view studies and give feedback

\section{Ankabut}

Ankabut is the UAE's Advanced National Research and Education Network (NREN), which offers academic institutions connectivity to other education networks around the world. In addition, Ankabut connects schools and public institutions across the UAE with an effective cost model and cooperates in the national, GCC regional and international arenas, representing the UAE at conferences, exhibitions and forums. It also offers QoS-based networks with IPv4/IPv6, multicast and advanced services on a "Closed Group Network" with "Public Interest Purposes."

Ankabut is very keen and ambitious in terms of providing nonproprietary software to the research and educational sectors in the UAE over its dedicated network. It must consider cross-platform issues, the high cost of licensing products and the adoption of OSS standards as a framework to deliver high quality services. Ankabut has shown a great deal of interest in collaborating with us on the proposed DVN project. They have shown their willingness to contribute, in terms of operational management, connectivity, deployment and data management, as well as providing a central data repository.

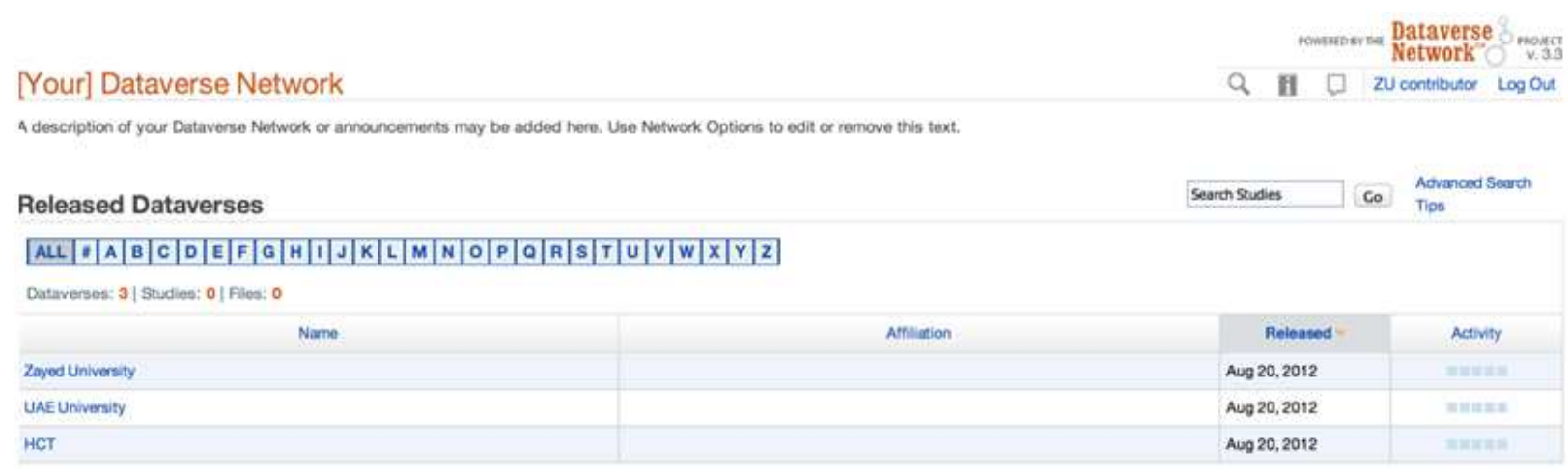

Fig. 1. The simple interface of our DVN design 
As Ankabut is building a National Data Center to host its research and educational activities, our DVN has been hosted by the Data Center and has been accessible to the development team.

\section{Research Design}

The National Research Foundation, along with other organizations in the UAE, are leading the initiative to integrate the efforts of social research institutions to support the decision making process in the UAE. During a forum on May 29, 2012, at the Emirates Center for Strategic Studies and Research, a number of eminent social science researchers raised the issue of the real need for a centralized repository for the researchers in this field in the UAE, which will enable them to share, connect, distribute, reference, extract and analyze research data. We described many OSS examples that could help researchers in the forum, demonstrating one in particular, the Dataverse Network (DVN), which could meet the need for a centralized. We then had a group of students from Zayed University deploy a dataverse on the DVN to serve as a repository and call it "Bayanatona", which means "Our Data" in English. The group went on to work with Ankabut to carry out this project and fulfill the mission of connecting social science researchers in the UAE. According to the survey and the interviews conducted among researchers, the contribution of Bayanatona could be vital to the improvement of technologies and support research and knowledge development in the field of social sciences in the UAE. Figure 2 summarizes our methodology, starting with a survey of researchers at various universities in the UAE in the cities of Abu Dhabi, Al Ain and Dubai. We then made the University Of Sharjah (UOS) the focus of a detailed case study and presented the challenges and recommendations of faculty members in the Department of Social Science at UOS. The final step in our methodology was to install the Bayanatona DVN on the Ankabut server and to organize training for social science researchers at UOS in the UAE.

\section{UAE University Overview}

The social sciences encompass various disciplines, including anthropology, archeology, business, communications, demography, economics, education, environmental design, geography, law, political science, psychology, public administration, public health, public policy, social work, sociology and urban planning, along with the off-shoots of these disciplines (Gerring, 2011). Researchers, practitioners and social scientists all agree on the enormous importance of social science research in advancing human knowledge and informing public policy makers (O'Connell, 2002). Social science research is an important resource for governments and various administrations, including that of the UAE, are aiming to improve the level of social science research in their countries. The largest and most-oil rich of the seven emirates of the UAE, Abu Dhabi, is expected to triple in population by 2030 and, as a result, social science research will have important implications for this country's vision, as well as providing relevant applications that will assist in its development. The culture of the UAE is one of the most talked about in social science research, as Arabic culture globally and the teachings of Islam in particular are often considered to be ideally represented in this country (Klein et al., 2009).

Academic libraries are used by institutions of higher learning to store and retrieve social science research data. They perform a myriad of functions and maintain their collections in both print and electronic format. Tega and Baro (2008), for example, examine how university libraries in Nigeria retrieve and store information about social science research. Their collections include books, newspapers, magazines, photographs, theses and dissertations, audiovisual materials, microforms, Compact Disk-Read Only Memory (CD-ROM), microfiche and microfilm readers and computers with CD-ROM drives. With advances in the area of information technology, libraries have increasingly adopted tools that have more efficient and effective ways to acquire, organize, store and disseminate information. The new information technologies have become critical components in shaping information systems for libraries. The use of computers and the Internet to perform literature searches have become common. University libraries now have international links and use network features to access a wider range of information. University libraries have also been converting their card catalogues into machinereadable forms and they have changed their emphasis from book collection and storage to providing access to electronic information services, as have other information-centered institutions (Tega and Baro, 2008; Tenopir, 2008; Nancarrow et al., 2008). Despite this, social science researchers in the UAE still face challenges researchingin their field. As we show in this study, we have collected feedback from social science researchers at universities in four main cities: Abu Dhabi, Al Ain, Dubai and Sharjah. The survey 
that we conducted reveals many similarities among these universities

\section{UOS Case Study}

According to Dr. Hussain Al Othman, the Sociology Department that he heads occupies a distinguished position both locally and regionally. With its highly qualified and experienced faculty, the department has undergone rapid development in the areas of research and community service and implemented the latest educational methods in its Bachelor's and Master's degree programs. He also states, "The Master's program in Applied Sociology is the first of its kind in the Arab world. The students in this program make up about $61.2 \%$ of the total number of students enrolled at the college. In accordance with our five-year strategy, the department intends to establish a doctoral program in this subject in the academic year 2013/2014." We decided that this department would be an ideal choice as the detailed case study for this study, enabling us to draw conclusions about the challenges of conducting research in the social sciences there and to elicit recommendations from its practitioners. The interviews we conducted among the faculty members in this department indicate that, although there are library resources available at UOS, researchers continue to face challenges similar to those indicated by their colleagues at other universities, revealing a need for a solution that serves all researchers in universities across the UAE. Details of our proposal for a solution are provided in the next section.

\section{The Bayanatona Project}

The plan for the Bayanatona project, which began in February, 2013, was simple, but needed to be carefully studied. The team had to find a quick, free and easy solution to the data repository problem and this was the dataverse network. The team also had to find a host, choose the best operating system for the installation and learn the technical aspects of the project. This plan is explained briefly below.

Ankabut was pleased to meet up with the team and discuss hosting the DVN. The meeting covered storage space, support and various technical aspects of the project. After the meeting, Ankabut agreed to host the DVN free of charge and offered the team an 8core CPU, 4 Gigabytes of RAM and 1 Terabyte of storage space.

In order to keep access to the DVN free of charge over the long term, an OSS operating system was the most desirable option. The Red Hat 6 operating system was the first to be tested, as it was the original operating system on which the DVNhad been developed. However, the team rejected this operating system because of the many difficulties encountered with its implementation. The second OSS operating system they tried was Ubunto 11. Ubunto ran the DVN successfully despite minor implementation errors and was soon running the project smoothly. The team also wanted to test the project on operating systems that are more familiar to users in the UAE, such as Windows7 and MacOS. Although these commercial operating systems had different systems of implementation, they could be used for our project. Among these options, the team chose Ubunto 11 as the operating system. Consultation with Ankabut about the operating system produced a new optionCentOS 6. CentOS 6 is an OSS operating system which, according to Ankabut, would offer a more stable environment. The team agreed to test the project on CentOS 6. It was, in fact, the most effective operating system for the Bayanatona project and was the ultimate choice.

\begin{tabular}{|l|l|}
\hline $\begin{array}{l}\text { UAE universities: } \\
\text { Overview }\end{array}$ & $\begin{array}{l}\text { - Forum at the emirates center for strategic studies and research } \\
\text { - Survey of universities in Abu Dhabi, Dubai and Al Ain }\end{array}$ \\
\begin{tabular}{|l} 
University Of Sharjah \\
(UOS) case study
\end{tabular} & $\begin{array}{l}\text { - Interviews } \\
\text { - Detailed survey of UOS faculty members }\end{array}$ \\
Dataverse Network (DVN) & $\begin{array}{l}\text { - Bayanatona project } \\
\text { - Collaboration with Ankabut }\end{array}$
\end{tabular}

Fig. 2. Research methodology for enhancing social science research in the UAE 


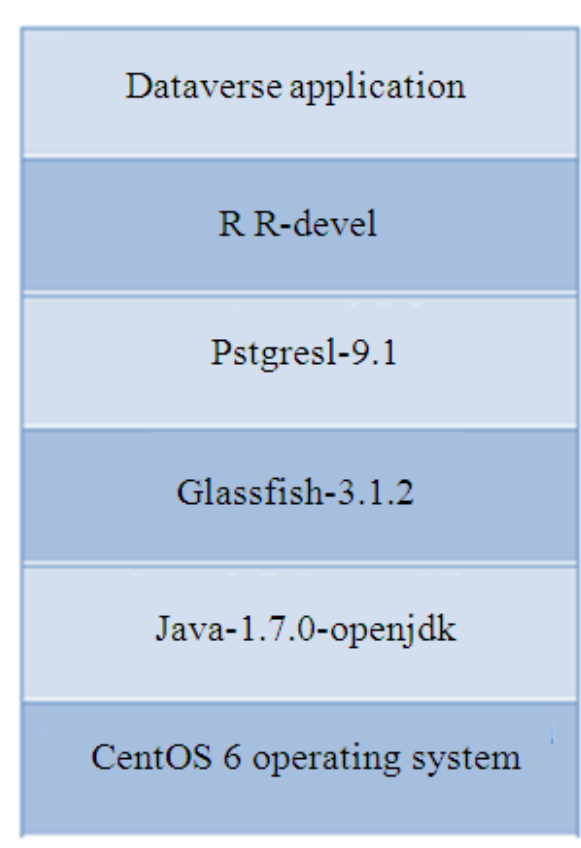

Fig. 3. Dataverse network tiers

The DVN is a multilayer application. There are four applications that must be installed before installing the dataverse application itself and these are: Java 1.7.0 open JDK, glassfish server 3.1.2 open Source Edition, Postgre SQL 9.1 and R R-Devel. The order of the layers, or tiers, has to be correct in order for the project to work. Figure 3 shows the tiers of our DVN. The top tier is the dataverse application. Java1.7.0 Open JDK is installed to support the run time of the Java environment programs. Glass Fish is an application server that helps to carry the DVN application. Postgre SQL is installed to manage databases created for research and R R-Devel handles statistics and graphs.

\section{Results}

The forum findings, the survey data analysis and the interviews showed more commonalities than differences in both the challenges and the recommendations.

\section{UAE University Results}

The forum was the starting point for integrating the efforts of social science research institutions to support the decision making process in the UAE. Many respected speakers and panelists were invited to speak on a number of topics in different sessions, such as: Challenges facing the integration of social science research efforts in the UAE; national priorities for scientific research in the field of social science in line with the goals of the UAE Government Strategy and the UAE Vision 2021; the use of modern technologies to serve researchers and institutions working in the area of social science research (an integrated social research activities database, social network for researchers, e-learning); and, last but not least, the best mechanisms to ensure communication and integration among stakeholders in social studies and social studies research in the UAE. Overall, communication is lacking among social science researchers in the UAE. According to Dr. Ateeq Jakka, associate professor and assistant dean of research at UAE University, the institution has more social science researchers and produces more research papers than any other university in the country. However, within the university, researchers are often unaware of their colleagues' research topics and outcomes. This lack of communication creates redundancies in research work. At the forum, Dr. Jakka strongly emphasized the importance of increasing communication among researchers by establishing a database that includes all work of the country's researchers. Many of the other speakers and panelists agreed with Dr. Jakka's recommendation and this was documented by the forum organizing committee.

According to the results of our survey, which was distributed to more than 140 social science researchers in four cities (Abu Dhabi, Al Ain, Dubai and Sharjah) in the UAE, more than $90 \%$ of the respondents maintain that they face challenges conducting research in their field in this country. Table 1 liststhe main challenges and recommendations on which there is agreement.

We also asked the survey participants about the resources available to them when they were conducting their social science research. Most of them indicated the following as their main resources:

- Libraries

- Books and other text books relevant to the UAE context

- Journal articles

- Government documents and statistics

- Personal communications with social work practitioners and social work managers in the field, senior students and UAE colleagues

- Interviews, questionnaires and scales

- Opportunities to participate in international conferences

- Online databases and OSS resources available on the Internet

- The Internet, although minimal relevant material was available 
Table 1. Challenges faced by survey respondents and their suggested for improvement

Challenges faced by social science researchers in the UAE

Suggestions to improve social science research in the UAE

No communication between social science researchers in the UAE

Lack of a centralized database to keep up-to-date research records.

- Not enough resources available.

- Not enough conferences and seminars in the UAE.

- No clear ethical policy regarding social science research.

- It is difficult for a non-Arabic speakers and expats from non-Arab countries to access resources in the form of real people prepared to share information (without collaboration with Arabic speakers and/or Emirati co-researchers, progressing with practical research is very difficult)

- It is difficult for Emirati researchers to obtain statistical or other information that seems to be under the control of highly placed government official

(Bureaucratic procedures may hinder research).

- Seek closer relationships with many social services and organizations in the UAE and develop research around the issues that really matter for building the UAE community.

- Provide more online resources on social science research to rein force the importance of the social sciences in every aspect of life. - Integrate the social sciences with other disciplines like engineering and technology to reap maximum benefits.

- Form professional bodies and ensure that they conduct programs in a timely fashion.

- Hold a conference or create an academic task force - Suggest large research projects and prioritize them.

- Solicit funds and assemble research teams to

design and conduct the proposed research projects.

- Provide awards for young researchers and acknowledge the development of new social science methodologies.

- Engage in international collaborations.

- Establish and maintain fair and ethical practices in conducting research.

- Offer Ph.D. programs in universities in the humanities and social sciences and develop the expert is e and resources required for these programs.

- Allow access to schools, universities.

for social science students to conduct their research.

It is clear that the DVN could be an affordable solution that responds to most of the suggestions proposed by the survey participants. Once this online repository has been established and any universities involved in this type of research can contribute their own individual dataverse and social institutions can connect to this application in the form of another dataverse, a professional body of research will be created that will be accessible to all. This will close the gap that now exists among the parties concerned and move research forward in a productive and collaborative way.

\section{UOS Results}

We conducted interviews with eight faculty members in the Department of Social Science at UOS to understand the challenges researchers face in greater depth.

\section{Social Science Research Challenges at UOS}

According to Dr. Hussain AlOthman, the head of the department, some research papers are difficult to obtain, which makes writing a literature review a challenge. For example, one of the journals in this field gives the university approximately $20 \%$ accessibility-which is clearly inadequate. A higher percentage of material can be obtained from the journal, but at a higher cost. The library does provide a certain number of research papers, although researchers are limited when it comes to obtaining papers from abroad. While it is true that interlibrary orders can be placed through the UOS library, there little similarity between the papers available and the topics being researched. Moreover, that availability is limited by cost and the papers ordered could take at least a month to arrive. For some databases, only a preview of a well-known paper is given and the complete paper must be purchased. In the case of temporary subscriptions, only limited data are provided. Dr. Al Othman also spoke of a lack of communication among researchers locally, which means that they may have limited knowledge of their peers' research. His conclusion is that finding research papers in the Middle East is extremely difficult without a database of researchers and their work.

Dr. Osama Abd El Bary points out that the four major factors that hinder the advancement of social science research in the Middle East in general and in the UAE in particular are: The nature of the society, lack of funding, politics and the inability of people to network. The use of the Internet as a means of communication can limit the participation of older professors who do not use a computer on a day-to-day basis and, consequently, may miss out on online conference invitations. By the time an invitation finally arrives by mail, the deadline for submission 
has often passed. Dr. El Bar indicates that invitations are also extended socially; for example, a colleague might inform a professor of an upcoming conference. Rather than attend or contribute to conferences to benefit society, some professors will attend to enhance social bonds or to get a paper published. Funding is also an issue in conference attendance. If a professor is not funded to participate in a specific conference, he may not attend.

Political issues also prove to be a limitation in the Middle East, due to instabilities resulting from the Arab Spring. The topics on a conference agenda may be limited.

The quality of the network linking professors at different universities, locally or internationally, is another issue, according to Dr. El Bary. To have access to a sufficiently large number of papers, it is important to forgevery strong connections with colleagues at other institutions.

Professor Ahmad Khettabi mentions another significant obstacle social science researchers must overcome, at UOS specifically, but around the world as well. He feels that research should be shared among institutions, not accessed by individuals in a database. Publishing under the umbrella of an institution protects the researcher's intellectual property. In Professor Khettabi's opinion, knowledge is a weaponit can elevate or destroy and so must be safeguarded. Moreover, because library resources are poor in the UAE, the only option is to go outside the country to access them. However, libraries do not deal with the outside world in accordance with international rules and connections. For this reason, he depends on his social connections with colleagues in other countries to obtain the research papers he needs.

Another faculty member, Dr. Omaima Abouelkheir, has a different perspective. As the mother of three children, she finds it hard to manage her many responsibilities and counts on her colleagues and connections to obtain research papers and inform her about the latest research outcomes put forward at the conferences they attend.

\section{Social Science Research and Arabic Language}

Dr. Makia Himat high lights the important issue of language preference when writing a research paper. A paper written in Arabic which could be published in an international journal, particularly in the social science field, is difficult to translate into English. She feels that this challenge should be discussed, as many of her colleagues have the same problem. In her words: "If there were a lexicon available, a sort of unified vocabulary, to help in the translation process that would be helpful. There are dictionaries for the social sciences, but it is time consuming to go through them."

Professor Khettabi publishes papers in Arabic, English and French. He confirms that language is an obstacle: "Since our literature isn't deep, rather it is very shallow; it has no scientific depth." Since Arab "minds" have emigrated to foreign countries, their papers are written in foreign languages. The qualitative talks take place abroad, because those Arab researchers are publishing abroad in English, French and other languages.

Dr. Fakir Al Gharaibeh, who works in the same department as Dr. Khettabi, publishes in both Arabic and English. He publishes more in English though, since publishing in Arabic journals takes time and feedback is poor. For example, if one of his articles is rejected by an Arabic journal, its reviewers provide few details or reasons for doing so. He receives good feedback from English and American journals, which helps him in his research. Nevertheless, he has published a book in Arabic.

\section{Statistical Tools and Citations}

Most faculty members at UOS use SPSS statistical software in their classes, although it is often an old version. They agree that using the old version does not hinder teaching. They stress that it is the job of the IT Center to make sure that subscriptions do not expire. Subscription difficulties arise because the IT Center is not diligent about renewals and must be constantly reminded. In one example, an expired subscription caused software to shut down in the middle of a lecture.

In general, faculty members cite references manually, which can take a few days to organize in are search paper.

\section{Conferences and Seminars in the UAE}

There are no problems with locating social science seminars and conferences in the UAE. They are organized in response to invitations from ministries and social work institutions.

According to Professor Nayef Odeh, there are enough of these events, but the nature of the academic work schedule makes attending them difficult; for example, exams act as a hindrance. Also, they may not work well with a researcher's schedule, as pressure at work and the desire to spend time with family make it difficult to connect with one's peers. 


\section{Recommendations for Improving Social Science Research at UOS}

Dr. Al Gharaibeh is on the editorial board of one of the leading journals in the social science field, International Social Work (ISW). This position and his history of study in Australia allow him access to many international journals in the field. He attends at least two conferences a year, which keeps him up to date with current research and produces at least one research paper per year on a social topic specific to the UAE. He has published two papers on divorce, one financially supported by the Marriage Fund and the other by the Scientific Research Fund. He has also received financial support from the Emirates Foundation. In addition, he has collaborated with researchers at other universities (i.e., UAE University, Ajman University), as well as government institutions (i.e., Dubai Police). This demonstrates the strength of his network, both nationally and internationally.

Although Dr. Al Gharaibeh manages his research work well, he also feels that the UAE is in need of a research repository. He maintains that, although we know that joint research is more effective than individual research, there is a lack of emphasis on joint research, noting that a team environment creates the opportunity for the exchange of expertise and views and ultimately a better analysis of the topic.

The chair of the department agrees with Dr. Al Gharaibeh'srecommendation: "The best solution is the existence of a database for articles in the Arab world in the social science field. If there's a database, problems will be solved."

\section{Discussion}

Innovations in computer technology that are applicable to social science research can be subdivided into four categories: Data analysis, word processing software, the virtual institution and electronic publication. In terms of data analysis, computer experts have developed various statistical and other data analysis software for qualitative and quantitative analytical techniques. Powerful world processors have been installed with sophisticated proofreading and bibliography management programs to make the writing and typesetting of research manuscripts easier. Advanced telecommunications technologies have made it possible and affordable to integrate a network without borders, which enables scholars to collaborate through a single virtual institution, regardless of their geographical location. Finally, data storage and retrieval technologies have given social science researchers the ability to immediately access almost any type of information they want (Burgess, 1992; Lahl and Pietrowsky, 2008). For example, some countries have their own system to actively manage digital content over time to ensure ongoing access, similar to the National Digital Information Infrastructure and Preservation Program (NDIIPP) led by the Library of Congress in the US (Ashendfelder, 2009).

The Dataverse Network (DVN) is an OSS application to publish, share, reference, extract and analyze research data. It is an online digital repository for social science research data (Crosas, 2011). It provides a central repository infrastructure for professionally archiving backups, data for recovery purposes and standards-based persistent identifiers, as well as addressing data fixity and metadata storage, conversion and preservation (Crosas, 2011). The DVN facilitates the availability of data to other researchers, which in turn enables those researchers to replicate the work of others. It is a complete computer technology solution for social science researchers which covers the four categories discussed earlier in this section. In addition, it overcomes all the challenges described by the invited speakers and panelists at the forum, as well as those pointed out by the survey and interview participants.

After testing the installation on several operating systems, the team finally decided to use CentOs 6 as the base operating system. After multiple attempts and the correction of a number of errors, the DVN project was successfully installed in the test environment and the team was confident enough to transfer the installation to the Ankabut server. This was done remotely using Putty and an SSH connection. The process took thirty minutes and no errors were found. The project remained unpublished and could only accessed locally for testing purposes, until November, 2013, at which time it became available for public use.

The Bayanatona Dataverse Network is a user-friendly application in terms of its flexibility and the ease with which it can be managed. The most important feature of Bayanatona is that it can accommodate multiple administrators, dataverses and users. The ability to assign multiple administrators to various sections of the DVN will help to manage the database as it grows, as they can fully control and customize their own section. They can also create sub-dataverses from the original dataverse, to include more categories and even authors. Finally, administrators can control what privileges are assigned to users. They can choose contribution methods and restrict users to the content of specific research, for example. 
Training sessions were delivered to eight faculty members in the social science department at UOS. Those faculty members experienced a high level of satisfaction. They agreed to upload their papers and be the first institution in the country to use the Bayanatona project. The feedback we received following the training sessions was very promising and included these comments: "If all social science researchers in UAE universities agree to upload their work, most of our research problems will be solved"; "This project is exactly what we are looking for"; and, "This project combines all the tools and features we need in one application and it will definitely ease the research process and makes it more promising. Finally, the team developed a user manual for other researchers interested in uploading their social science research to Bayanatona and Ankabut has posted it on their website. Bayanatona has now been added to Ankabut to serve academic institutions in the UAE.

\section{Conclusion}

In this study, we investigated the challenges faced by social science researchers in four main cities in the UAE: Abu Dhabi, Al Ain, Dubai and Sharjah. As a result, the Bayanatona Dataverse Network, an OSS application, was proposed as a complete solution for these researchers in the UAE. It overcomes most challenges that were described by the invited speakers and panelists at the forum, as well as those highlighted by the survey and interview participants.

The promising results we acquired in our research suggest that future research should include three major objectives: (1) To encourage researchers at other universities in the UAE to upload their work to Bayanatona and conduct a survey on their application usage; (2) to encourage researchers at other universities in the Gulf and Arab countries to upload their work to bayanatona and conduct a survey on their application usage; and (3) to collaborate with the team at harvard University to connect our dataverse to their dataverse and then examine the possibility of creating an Arabic interface for the dataverse network.

\section{Acknowledgment}

The authors thank Mr. Fahem Al Nuaimi, Chief Executive Officer of Ankabut and Dr. Ahmed Dabbagh, Manager of Technology and Services Development for Ankabut for their generous support of this research paper. We are also grateful to the group of Zayed University students for implementing the project on the Ankabut network and to the social science researchers at Zayed University, University of Sharjah, Abu Dhabi University, United Arab Emirates University and the Birla Institute of Technology and Science for contributing to the survey study.

\section{Author's Contributions}

All authors equally contributed in this work.

\section{Ethics}

This article is original and contains unpublished material. The corresponding author confirms that all of the other authors have read and approved the manuscript and no ethical issues involved.

\section{References}

Ashendfelder, M., 2009. NDIIPP models for mass data transmission and storage. Library Tends, 57: 541-555. DOI: $10.1353 /$ lib.0.0051

Burgess, G., 1992. Telecommunications, information storage and retrieval and peace and change research. Peace Change, 17: 6-12.

DOI: $10.1111 / \mathrm{j} .1468-0130.1992 . t b 00165 . x$

Crosas, M., 2011. The dataverse network $\AA$ : An opensource application for sharing, discovering and preserving data. DLib Magazine, 17: 1-9. DOI: $10.1045 /$ january2011-crosas

ESCWA, 2011. National profile of the information society in the United Arab Emirates. New York.

Gerring, J., 2011. Social Science Methodology: A Unified Framework. 2nd Edn., Cambridge University Press, New York, ISBN-10: 1139503774, pp: 495.

Jaspersoft, 2010. The state of enterprise open source software after the oracle acquisition of sun Microsystems.

Klein, A., M.F. Waxin, E. Radnell, 2009. The impact of the Arab national culture on the perception of ideal organizational culture in the United Arab Emirates: An empirical study of 17 firms. Edu. Bus. Society, 2: 44-56. DOI: 10.1108/17537980910938479

Lahl, O. and R. Pietrowsky, 2008. Tracer: A generalpurpose software library for logging events in computerized experiments. Behavior Res. Meth., 40: 1163-1169. DOI: 10.3758/BRM.40.4.1163

Nancarrow, C., J. Pallister and I. Brace, 2008. A new research medium, new research populations and seven deadly sins for internet researchers. Qualitative Market Res., 4: 136-149. DOI: $10.1108 / 13522750110393044$

O'Connell, J., 2002. Wanted: Gifted students in social sciences. Social Studies Review. 
OSI, 2013. Retrieved on October 10, 2013.

Tega, R.E. and E.E. Baro, 2008. Patterns of Information storage and retrieval in university libraries in Nigeria. Library Hi Tech. News, 25: 19-26. DOI: $10.1108 / 07419050810877526$

Tenopir, C., 2008. Online systems for information access and retrieval. Library Trends, 56: 816-829. DOI: $10.1353 /$ lib.0.0005
UAEITR, 2013. United Arab Emirates Information Technology Report Q1. Business Monitor International, United Arab Emirates.

WEF, 2013. The global information technology report. World Economic Forum, Soumitra Dutta, Cornell University. 